ARTICLE

Received 20 Dec 2012 | Accepted 21 May 2013 | Published 19 Jun 2013

DOI: $10.1038 /$ ncomms3039

OPEN

\title{
Exploring the MHC-peptide matrix of central tolerance in the human thymus
}

\author{
Eleni Adamopoulou', Stefan Tenzer², Nina Hillen ${ }^{3}$, Paula Klug${ }^{1}$, loanna A. Rota1', Silvia Tietz ${ }^{1}$, Madlen Gebhardt ${ }^{1}$, \\ Stefan Stevanovic ${ }^{3}$, Hansjörg Schild ${ }^{2}$, Eva Tolosa ${ }^{4}$, Arthur Melms ${ }^{1, \dagger} \&$ Christina Stoeckle ${ }^{1, \dagger}$
}

Ever since it was discovered that central tolerance to self is imposed on developing T cells in the thymus through their interaction with self-peptide major histocompatibility complexes on thymic antigen-presenting cells, immunologists have speculated about the nature of these peptides, particularly in humans. Here, to shed light on the so-far unknown human thymic peptide repertoire, we analyse peptides eluted from isolated thymic dendritic cells, dendritic cell-depleted antigen-presenting cells and whole thymus. Bioinformatic analysis of the 842 identified natural major histocompatibility complex I and II ligands reveals significant crosstalk between major histocompatibility complex-class I and II pathways and differences in source protein representation between individuals as well as different antigen-presenting cells. Furthermore, several autoimmune- and tumour-related peptides, from enolase and vimentin for example, are presented in the healthy thymus. 302 peptides are directly derived from negatively selecting dendritic cells, thus providing the first global view of the peptide matrix in the human thymus that imposes self-tolerance in vivo.

\footnotetext{
${ }^{1}$ Department of General Neurology, Hertie Institute for Clinical Brain Research, 72076 Tübingen Germany. ${ }^{2}$ Institute for Immunology, University Medical Center of the Johannes Gutenberg University of Mainz, 55131 Mainz, Germany. ${ }^{3}$ Department of Immunology, Institute of Cell Biology, University of Tübingen, 72076 Tübingen, Germany. ${ }^{4}$ Institute for Immunology, University Medical Center Hamburg-Eppendorf, 20246 Hamburg, Germany. †Present addresses: Department of Neurology, University of Erlangen-Nuremberg, Erlangen, Germany (A.M.); Institute of Pharmacology, University of Bern, Bern, Switzerland (C.S.). Correspondence and requests for materials should be addressed to C.S. (email: christina.stoeckle@web.de).
} 
A functional immune system must be able to effectively recognize and fight pathogens while staying tolerant to the body's own components. This is achieved by central and peripheral tolerance mechanisms. Central tolerance to self is imposed on $\mathrm{T}$ cells during their development in the thymus, where they are educated to be self-referential, recognizing peptides bound to self-major histocompatibility complex (MHC) molecules, as well as self-tolerant, meaning that they remain unresponsive to self-peptides displayed on the very same MHC molecules ${ }^{1,2}$.

In order to fulfil both criteria, the $\alpha \beta$ T cells must pass through two major checkpoints in their development, which are controlled by antigen-presenting cells (APCs) in distinct thymic microenvironments. These APC comprise cortical (cTECs) and medullary thymic epithelial cells (mTECs), as well as both resident and immigrant dendritic cells (DCs). During development, thymocytes must rearrange their genes in order to express a functional T-cell receptor (TCR) that can successfully interact with self-peptide-MHC ligands on cTEC, from which they will then receive a survival signal (positive selection). Positively selected thymocytes migrate into the thymic medulla, where they interact with self-peptide-MHC complexes displayed by mTECs and DCs. T cells expressing a high affinity TCR for these complexes will be negatively selected and undergo apoptosis. Only T cells with a low/intermediate affinity TCR for selfpeptides will be allowed to leave the thymus as naive or regulatory $\mathrm{T}$ cells ${ }^{1,2}$. If a $\mathrm{T}$ cell that strongly recognizes self-peptides fails to be deleted, for example, because the corresponding self-protein is not expressed in the thymus or the epitope is destroyed during antigen processing, autoreactive $\mathrm{T}$ cells can escape into the periphery, where they can be activated and cause autoimmune disease $^{3}$. Therefore, the interaction between the TCR of developing thymocytes and the MHC peptide ligandome of distinct thymic APCs is crucial for shaping the T-cell repertoire and central tolerance.

Myeloid DCs (mDCs) in the thymus were the first cells discovered to be responsible for deletion of autoreactive $\mathrm{T}$ cells in the thymus and are crucial for maintaining tolerance to self ${ }^{4,5}$. Although, in contrast to mTECs, they are not specialized in expressing tissue-specific antigens such as proinsulin, they can efficiently capture these from mTECs and present them to developing $\mathrm{T}$ cells, inducing their deletion, a process that involves autoimmune regulator expression (AIRE) in $\mathrm{mTECs}^{6,7}$. A role of plasmacytoid DCs (pDCs) in negative selection has only been described very recently ${ }^{8}$ and thymic $\mathrm{B}$ cells might also be able to contribute to negative selection ${ }^{9}$. Although so far there is no direct proof that the different subsets of thymic APCs present different sets of peptides, there are two strong lines of indirect evidence supporting this notion. First, the different APCs express different sets of self-proteins. In addition to constitutively expressed house-keeping proteins and cell-type-specific proteins, mTECs express tissue-restricted antigens such as insulin under the control of the AIRE transcription factor ${ }^{10,11}$. Also, blood-born $\mathrm{mDCs}$ and $\mathrm{pDCs}$ can import peripheral antigens into the thymus where they delete self-reactive thymocytes $^{8,12}$. Second, both the MHC I and II antigenprocessing machinery differs between different thymic APCs, which should influence the epitopes that are generated ${ }^{13-16}$. The different APCs thus contribute to the diversity of the immunological self displayed in the thymus shaping the characteristics of the mature T-cell repertoire of each individual.

Our current knowledge regarding the self-peptide repertoire in the thymus derives mostly from studies in TCR transgenic mouse models, where expression of a particular antigen is a prerequisite for positive/negative selection. However, none of these studies provides evidence that the respective peptides actually contribute to negative selection under physiological conditions. In the early nineties, Marrack et al. ${ }^{17}$ successfully identified $\sim 20$ MHC IIbound peptides in pooled thymi of several hundred mice and a few natural ligands for positive selection of mouse OT-I cells are known ${ }^{18,19}$. More recently, another group has eluted peptides from whole-mouse thymus, using bioinformatics to narrow down on likely MHC I ligands ${ }^{20}$. In humans, presentation of an epitope from proinsulin on thymic DCs has been reported using monoclonal antibodies ${ }^{21}$, but beyond this, the human thymic peptide repertoire is unknown.

In this work we set out to explore the human thymus MHC ligandome. We describe an in depth analysis of the peptide repertoire presented by both MHC class I and class II molecules in the healthy human thymus, both for whole thymus as well as isolated thymic mDCs. In summary, we here present the first data on naturally occurring peptides on which human $\mathrm{T}$ cells are selected under physiological conditions, including peptides from DCs which are responsible for deletion of autoreactive $\mathrm{T}$ cells.

\section{Results}

Identification of peptides presented by thymic APCs. To address the question which peptides are naturally presented to developing thymocytes to induce negative selection under physiological conditions in humans, we analysed peptides eluted from both MHC I (pan-class I human leucocyte antigen; HLA) and II (HLA-DR) complexes from isolated thymic mDCs by mass spectrometry and compared them with the repertoire of the MHC-bound peptides eluted from cells of the APC-enriched, mDC-depleted (containing pDCs, TECs and other thymic APCs, that is, ligands for both positive and negative selection) as well as whole-thymus fractions (Fig. 1).

One major challenge for MS-based analysis of peptides presented by thymic APCs is their low frequency and their purification in sufficient numbers to allow ligandome analysis. Typically, the number of cells needed for such experiments is in the order of $10^{8}$, limiting the possibilities for analysis of primary purified cell types. In our experiments, we identified a total of 842 peptides from the different cell preparations of the eight individual thymi investigated, of which 621 were eluted from MHC I and 221 from MHC II molecules. In the individual mDC preparations, we successfully identified up to 90 peptide sequences eluted from MHC I molecules and up to 48 from MHC II from as little as $30 \times 10^{6}$ cells from four different donors (Table 1). Complete lists of the peptide sequences obtained from the different samples and their source proteins are given in Supplementary Data 1.

Cross-talk between MHC I and MHC II pathways. Peptides presented by MHC I and II derive from different cellular compartments. Typically, nuclear and cytosolic proteins are presented by MHC I, while proteins that are present in the vesicular compartments or the cell membrane or are taken up from the extracellular environment are presented by MHC II molecules. However, especially in professional APCs, there is considerable cross-talk between those two pathways ${ }^{22}$. Mechanisms by which cytosolic/nuclear proteins gain access to the MHC II pathway include autophagy and uptake of apoptotic cell ${ }^{23,24}$. Vice versa, proteins or peptides can shuttle out of the lysosome into the cytosol and thus gain access to the MHC I pathway ${ }^{22}$. In addition to this intracellular cross-talk, DCs may take up antigens from other cells such as mTECs, further broadening their peptide repertoire ${ }^{25,26}$.

To gain insight into the origin of the presented peptides as well as the dynamics of antigenic cross-talk between MHC presentation pathways in thymic mDCs and non-mDC APCs, we 


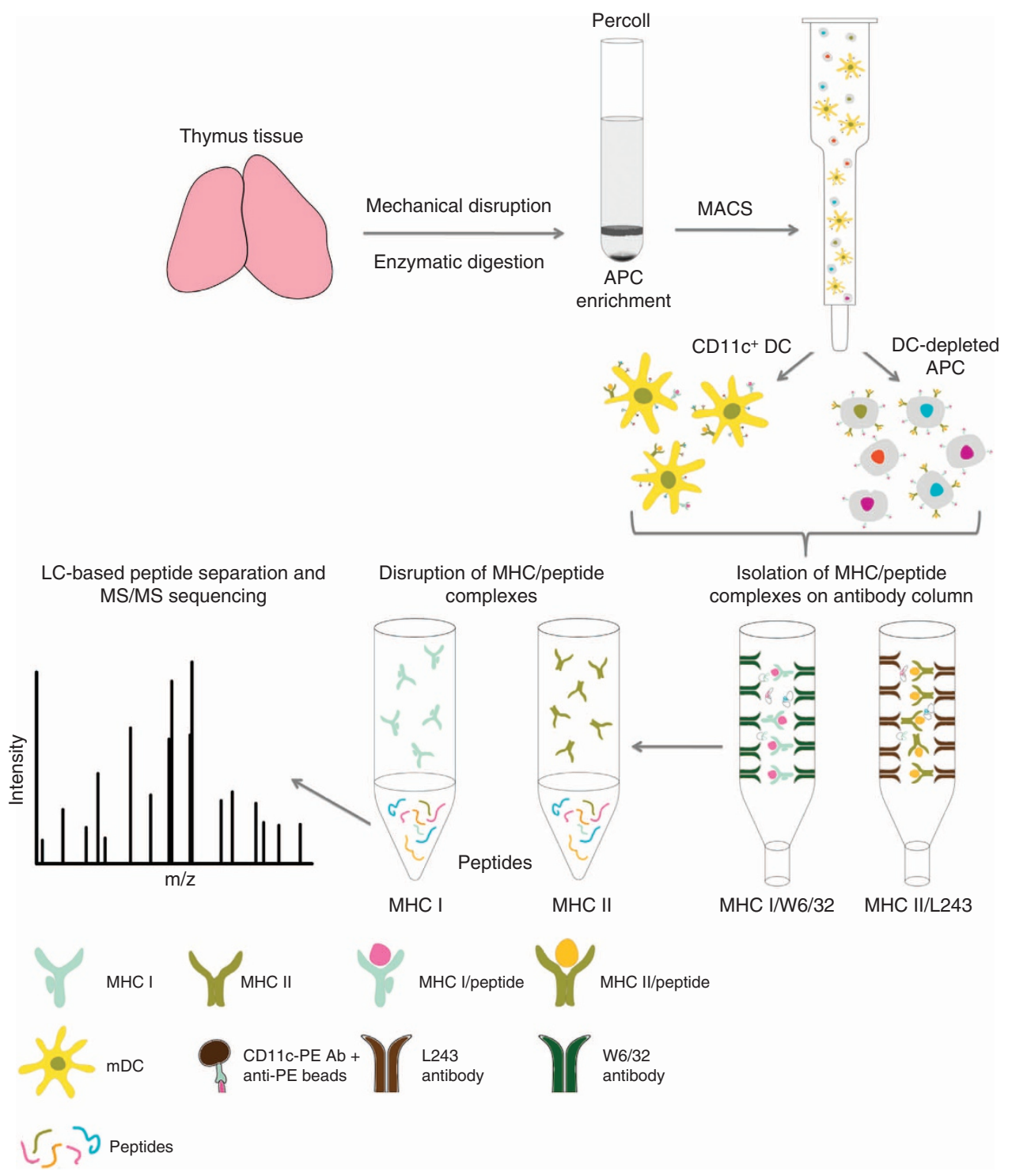

Figure 1 | Workflow diagram for the isolation of MHC I and MHC II ligands from CD11c ${ }^{+}$mDC and the DC depleted, APC-enriched fractions.

\section{Table 1 | MHC I and MHC II ligands identified on thymic CD11c $^{+}$dendritic cells of individual donors.}

\begin{tabular}{llcc}
$\begin{array}{l}\text { Tissue } \\
\text { number }\end{array}$ & $\begin{array}{c}\text { DC cell } \\
\text { number }\end{array}$ & $\begin{array}{c}\text { No. of MHC I } \\
\text { ligands }\end{array}$ & $\begin{array}{c}\text { No. of MHC II } \\
\text { ligands }\end{array}$ \\
\hline TT222 & $36 \times 10^{6}$ & 43 & 47 \\
TT251 & $66 \times 10^{6}$ & 90 & 35 \\
TT259 & $33 \times 10^{6}$ & 15 & 19 \\
TT260 & $30 \times 10^{6}$ & 29 & 24 \\
\hline
\end{tabular}

DC, dendritic cell; MHC I, major histocompatibility complex I; MHC II, major histocompatibility complex II.

The table contains the number of identified MHC I- and MHC II-derived peptides, the source tissue and the number of isolated DCs from which they were eluted.

performed automated database analysis supplemented by manual annotation of the source proteins and their subcellular localization. Depending on their topological origin, proteins were assigned either to the classical MHC class I (cytosolic, nuclear) or the MHC class II (extracellular, secretory, endocytic, and cell membrane) pathway, the mitochondrial and the ER compartment (Fig. 2a). As expected, the majority of MHC I ligands presented by both DCs and non-DC APCs derived from cytosolic/nuclear proteins, that normally do not intersect the endocytic compartment, such as histones, cytoskeletal proteins (actin, tubulin and fascin), metabolic enzymes (protein phosphatase 1, hydroxypyruvate reductase), and cytosolic chaperones (heat shock proteins). MHC class II-bound peptides originated from proteins present in different cellular compartments, including the extracellular milieu, the plasma membrane and the vesicular compartment with membrane proteins being the most abundant peptide generators (mostly from MHC class II proteins) in agreement with previous studies with other cell types ${ }^{24}$. A substantial number of MHC II presented peptides originated from blood or serum proteins, such as serum albumin, haemoglobin and apolipoprotein A2, providing direct evidence that thymic DCs sample the blood stream.

Interestingly, a considerable fraction of the MHC II ligands derived from source proteins localized to the cytosol or nucleus (35.4\% in DCs and $21.8 \%$ in non-DCs), which are topologically separated from the MHC class II pathway. Similarly, $22.4 \%$ of MHC I ligands on DCs derived from the vesicular/extracellular 

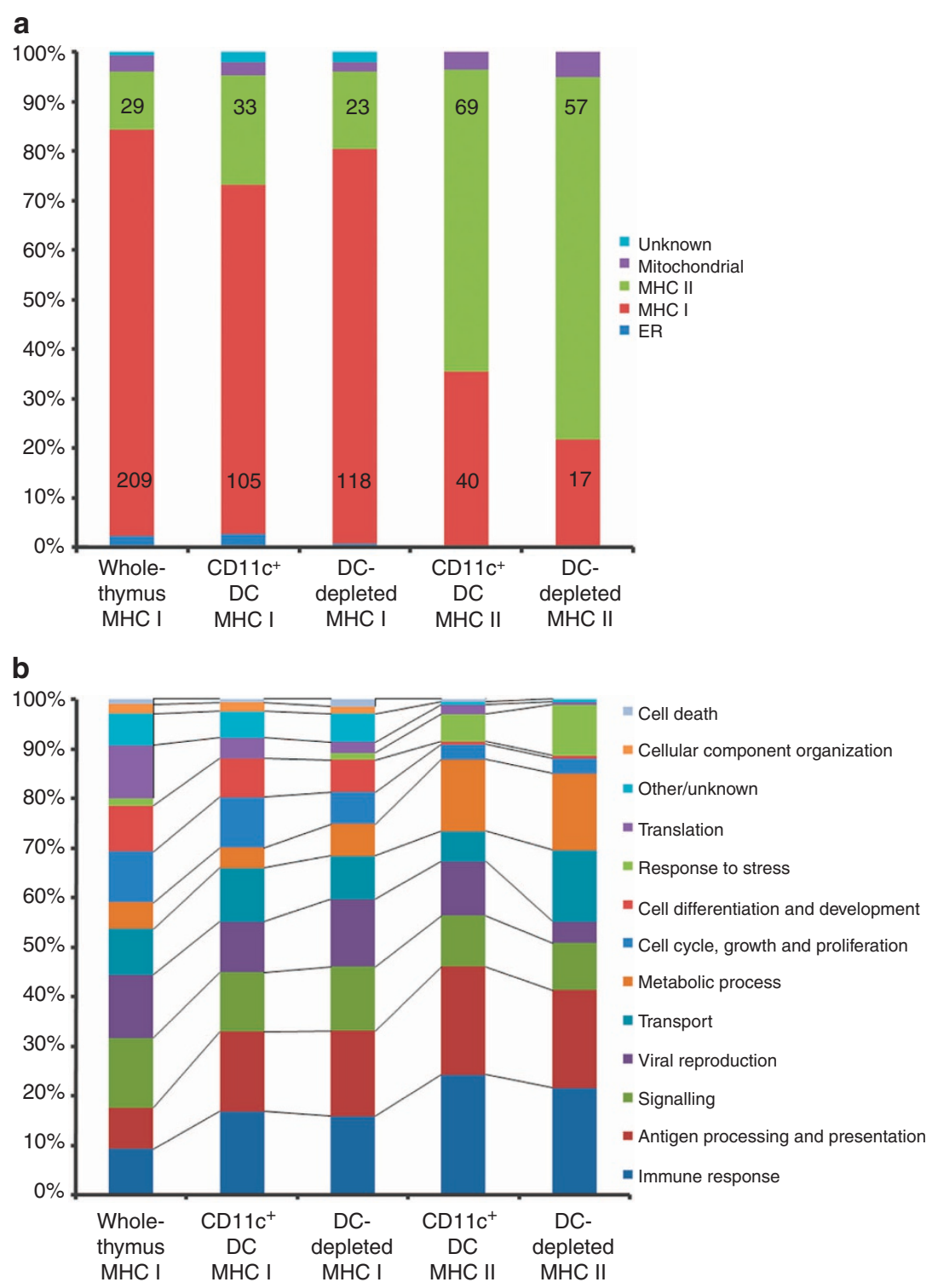

Figure 2 | MHC pathway assignment and functional categorization of the identified source proteins isolated from the different thymic sample preparations. The cellular localization (a) and biological processes (b) of the protein categories represented graphically as percentages are based on $n=$ the number of the individual proteins identified in each thymic APC preparation.

compartment (compared with $15.5 \%$ in the DC-depleted fraction and $11.4 \%$ in the complete thymus samples). Peripheral DCs are highly efficient at cross-presenting endocytosed antigens on $\mathrm{MHC}$ I and cytosolic/nuclear antigens on MHC II. It is therefore crucial to induce central tolerance also to cross-presented self-proteins. The high degree of cross-talk between the two pathways that we observe in thymic DCs, as well as to a lesser extent in the DC-depleted fraction, reflects this need to avoid peripheral autoimmune responses by deleting $\mathrm{T}$ cells that recognize cross-presented self-proteins.

A recent study has underscored the importance of autophagy for MHC II presentation during central tolerance induction. In mice, genetic interference with autophagy leads to an altered selection of certain $\mathrm{CD} 4{ }^{+} \mathrm{T}$-cell specificities that give rise to an autoreactive T-cell repertoire ${ }^{27}$. Most of the studies so far have addressed the in vivo relevance of autophagy mainly in TECs, and not thymic DCs, but in peripheral DCs autophagosomes have been shown to fuse with the MHC II loading compartment ${ }^{23}$. Our peptide data show considerable presentation of peptides derived from nuclear/cytosolic as well as some mitochondrial proteins on MHC class II by thymic mDCs, suggesting a role for autophagy in MHC II ligands sampling in these cells. Alternatively, these proteins could enter the MHC II pathway by uptake from other cells such as apoptotic thymocytes or live/dying mTECs m $^{7,28,29}$ or transfer of peptide-MHC complexes from mTECs ${ }^{30}$.

Nature of DC-presented peptides. Analysis of the biological processes associated with the peptides' source proteins revealed a prominent fraction of proteins participating in immune response' and 'antigen processing/presentation', biological functions reflecting the nature of the thymus as an immune organ (Fig. 2b). The most highly represented class of proteins in these groups were HLA molecules and, not surprisingly, their presence was more pronounced in the DC and DC-depleted samples (enriched for other thymic APCs), compared with whole-thymus samples. Most of the remaining class I and II natural ligands derive from house-keeping proteins (signalling, 
translation, metabolism, cell cycle and proliferation). Interestingly, peptides derived from proteins associated with metabolic processes are preferentially presented on MHC class II molecules, and peptides derived from proteins involved in cell cycle, growth, proliferation and differentiation are more abundantly presented on MHC class I molecules. The explanation for the preferential presentation of peptides derived from proteins involved in these processes by MHC I is that in these groups the majority of peptides derive from nuclear and cytosolic proteins involved in maintaining cellular structure (for example, the cytoskeleton) and transcription. These are typically degraded by the proteasome and presented via the classical MHC I pathway ${ }^{22}$. A closer look at the metabolic processes-associated proteins revealed that many of the MHC II-bound identified peptides in this group derived from cytosolic proteins. The sources of these peptides included a considerable number of long-lived constitutive metabolic enzymes like glyceraldehyde 3-phosphate dehydrogenase and phosphoglycerate kinase 1 . Interestingly, long-lived proteins have been suggested to be disposed by autophagic rather than proteasomal protein degradation ${ }^{31}$. Autophagic degradation of cellular material has been shown to significantly contribute to the peptide pool presented by MHC II (refs 23,24). In agreement with our results and in support of this idea, glyceraldehyde 3-phosphate dehydrogenase was previously identified as a source of natural MHC class II, but not class I ligands ${ }^{24,32}$. Hence, autophagic degradation of long-lived metabolic proteins might explain why these cytosolic proteins are presented by MHC class II rather than class I. The observed wide cellular functional distribution of proteins reflects the wide range of proteins that serve as a matrix for self-protein presentation in the thymus.

Peptides differ between individuals and APC. It has been suggested that different types of thymic APC present different nonoverlapping set of self-proteins, despite the existence of pathways allowing protein/peptide transfer between cells and despite the fact that a lot of basic cellular components like metabolic enzymes, and structural proteins should be expressed at similar levels in most cells. One reason for this might be the differences in the antigen-processing machinery between the different cell types $^{13-16}$. To address this question, we compared the source proteins of peptides presented on DCs to that of the DC-depleted fraction (enriched for other thymic APCs, for composition see Supplementary Table S1 and Supplementary Fig. S1). In total, we identified 302 peptides originating from 187 different proteins on DCs and 265 peptides from 184 different proteins in the DCdepleted fraction, 71 of which were present in both fractions (Fig. 3a). These include proteins such as HLA molecules, histones, proliferating cell nuclear antigen, actin, pyruvate kinase, serum albumin and $\beta 2$ microglobulin. Although, $\sim 1 / 3$ of the protein pool was shared between DCs and non-DC cells, the majority of the identified proteins were unique for each group, supporting the idea that different thymic APCs present different peptides.

Data from whole thymus indicated a large degree of variability of the source proteins from which peptides were presented in different donors (Supplementary Fig. S2), probably partially due to differences in HLA-binding preferences and protein expression. Quantitative differences in expression levels of individual MHC-peptide complexes might also play a role as our experimental approach preferentially detects high abundance peptides, especially when source material is limiting. To exclude the possibility that the limited overlap observed was due to issues of technical reproducibility rather than bona fide biological variability, we performed extensive validation experiments which showed that technical reproducibility was in the order of $85 \%$, while the overlap between samples derived from different donors was much lower (Supplementary Fig. S3), demonstrating that the differences observed were indeed due to biological, and not technical, variability.

To clarify whether this variability was also observed for the self-proteins directly involved in negative selection of $\mathrm{T}$ cells, we compared the source proteins of the peptides eluted from thymic DCs from different donors (Fig. 3b). Of the 187 proteins whose peptides were presented by $\mathrm{mDCs}$, only 27 were detected in more than one donor, and only one (HLA-DRB1) was shared by the four individual thymic DC samples. Peptides from seven proteins (HLA-DRA, serum albumin, tubulin $\beta 2 \mathrm{~A}$ chain, serine/ threonine-protein phosphatase PP1, astrocytic phosphoprotein PEA-15, bax inhibitor 1, signal transducer and activator of transcription $1-\alpha / \beta$ ) could be detected in three out of four DC samples, but peptides from the vast majority of proteins (160) were only detected in one sample, reflecting a high interindividual variability in the peptide matrix for negative selection. A similar picture is observed in the DC-depleted-derived peptide pool (Fig. 3c).

To further explore the differences between the HLA ligandome of DCs and non-DCs, we directly compared the source proteins (Fig. 4a) and the protein-derived peptides (Fig. 4b) that are present on the respective fractions from the same donors, eliminating the contribution of HLA-binding preference to peptide variability between fractions. Interestingly, only about a fifth of the detected proteins were presented by both DC and nonDC APCs, and for donor TT259 the overlap was even smaller. In summary, we demonstrate a high inter-individual variability of the MHC ligandome and furthermore, our results agree with the notion that different, but overlapping spectra of self-proteins on different thymic APCs results in partially non-redundant contributions of different APCs to negative selection. a

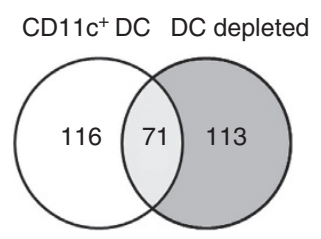

b

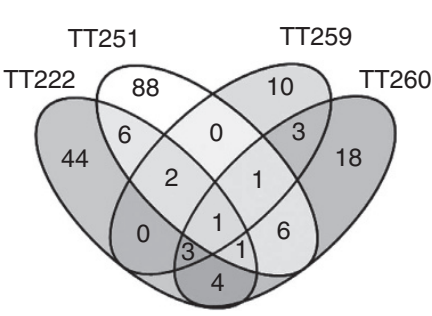

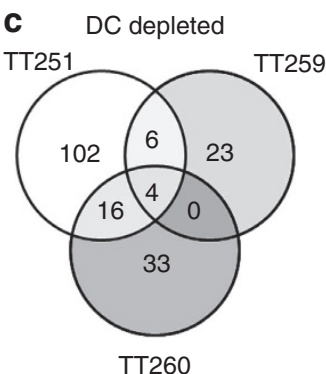

Figure 3 | Inter-individual variation in presentation of peptides from different source proteins presented by MHC I and II. The diagrams depict the number of different source proteins identified in the different cell preparations as well as overlap between samples. (a) Overlap of identified proteins between $\mathrm{mDC}$ and $\mathrm{mDC}$-depleted (enriched for other APC) fractions (individual donors taken together). Inter-individual variations of proteins presented on (b) DC of four different donors and (c) DC-depleted fractions of three individuals, respectively. 
T-cell epitopes linked to autoimmunity. Incomplete representation of the full spectrum of self-proteins (the immunological self) on MHC molecules of mTECs and DCs will result in a lack

a
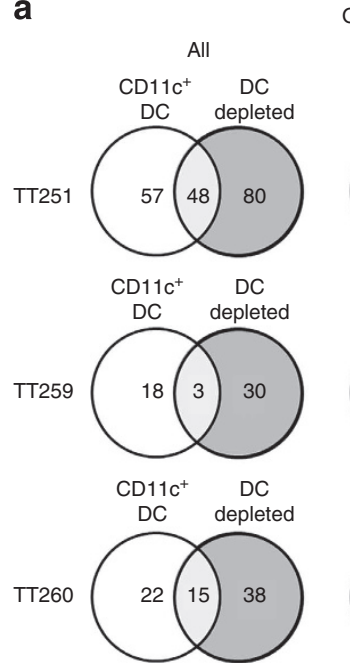

b

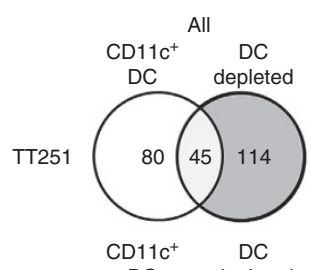

Overlap of presented peptides
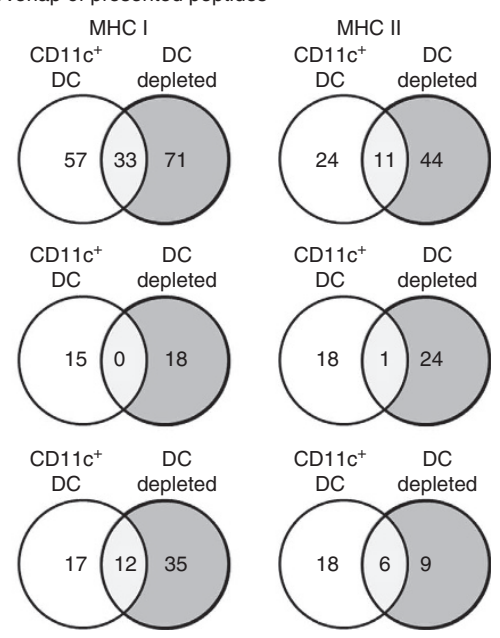

Figure 4 | Comparison of $\mathrm{mDC}$ and non-mDC fractions in each donor. Overlap of (a) source proteins and (b) presented peptides between $\mathrm{mDC}$ and $\mathrm{mDC}$-depleted cells in three individual thymi. of central tolerance to those epitopes that are not displayed, increasing the propensity for autoreactivity among mature $T$ cells in the periphery ${ }^{1,33}$.

We were therefore particularly interested whether we could identify peptides corresponding to known T-cell epitopes that have been described as targets in human autoimmune diseases. Literature search yielded a number of T-cell autoantigens that were also detectable in our samples (Table 2). The table only includes T-cell autoantigens for which reactivity has been experimentally verified in humans, such as vimentin (rheumatoid arthritis, sarcoidosis) ${ }^{34,35}$, ATP synthase (sarcoidosis) ${ }^{35}$, enolase 2 (multiple sclerosis) ${ }^{36}$ and heat shock protein 70 (type 1 diabetes mellitus $)^{37}$. For those autoantigens where reactivity against specific epitopes (rather than whole protein) was reported, the epitopes were identical to, or contained within the peptide sequences that we identified in our samples, mostly on DCs. This clearly demonstrates presentation of these epitopes in normal human thymus, which should lead to deletion of autoreactive $\mathrm{T}$ cells. We therefore hypothesize that in patients with autoimmune disease, presentation of these epitopes could be impaired, resulting in failure of central tolerance towards these epitopes.

Tumour-associated antigens in the thymus. Central tolerance is a double-edged sword. Although it protects us from autoimmune disease, it can also impede anti-tumour immunity. It is likely that presentation of self-proteins which tolerize the T-cell repertoire to self-antigens in order to avoid autoimmunity may also negatively impact the ability of $\mathrm{T}$ cells to mediate tumour immunity. This prediction has been confirmed by studies demonstrating the expression of tumour-associated antigens, including differentiation antigens and cancer-germline antigens, in human mTECs ${ }^{38}$, and moreover, expression of the human carcinoembryonic antigen (CEA), in mTECs of transgenic mice induces central tolerance ${ }^{39}$. A thorough literature search on natural MHC ligand repertoires in tumours revealed that a significant number of the MHC-bound peptides detected in our experiments are also found to be (over)presented in renal cell carcinomas ${ }^{40,41}$ or other disease tissues (Supplementary Data 2), including the SET nuclear oncogene and APOL1. Our findings provide an explanation why only low-avidity $\mathrm{T}$ cells against many cancer-associated antigens are present in the periphery ${ }^{42}$, as presentation of tumourassociated antigens by negatively selecting cells of the thymus should result in the deletion of high-avidity $\mathrm{T}$ cells. Therefore, even if they are found to be overexpressed on tumour tissues, they might not be the first choice as a target for immunotherapy. An

Table 2 | T-cell autoantigens/epitopes in humans identified in different thymus samples.

\begin{tabular}{|c|c|c|c|c|c|c|}
\hline Autoantigen & Epitope (thymus) & Thymus fraction & Disease & Epitope used in T-cell assay & Assay type & Ref. \\
\hline Vimentin & RETNLDSLPL (424-433) & DC & Rheumatoid arthritis & LPNFSSLNLRETNLDSLPL (415-433) & $\begin{array}{l}\text { ICS } \\
\text { IFN- } \gamma\end{array}$ & 34 \\
\hline $\begin{array}{l}\mathrm{H}^{+} / \mathrm{K}^{+} \text {ATPase } \\
\text { (ATP synthase) }\end{array}$ & $\begin{array}{l}\text { VPPVQVSPLIKLG } \\
\text { RYSAL (2-19) }\end{array}$ & DC depleted & Sarcoidosis & VPPVQVSPLIKLGRYSAL (2-19) & $\begin{array}{l}\text { ELISPOT } \\
\text { IFN- } \gamma\end{array}$ & 51 \\
\hline$\beta$-actin & RVAPEEHPVL (95-104) & DC & Sarcoidosis & NELRVAPEEHPVLLT ${ }_{(92-106)}$ & $\begin{array}{l}\text { ELISPOT } \\
\text { IFN- } \gamma\end{array}$ & 51 \\
\hline Heat shock protein 70 & $\begin{array}{l}\text { NVLRIINEP } \\
\text { TAAAIA }_{(168-182)}\end{array}$ & $\begin{array}{l}\text { DC } \\
\text { DC depleted }\end{array}$ & Type 1 diabetes mellitus & GLNVLRIINEPTAAAIAYGL (166-185) & ${ }^{3} \mathrm{H}$-thymidine & 37 \\
\hline Enolase 2 & IVGDDLTVTNPK & DC & Multiple sclerosis & Whole protein & ${ }^{3} \mathrm{H}$-thymidine & 36 \\
\hline Cytokeratin 17 & RLASYLDKV (125-133) & Whole thymus & Psoriasis & RLASYLDKV (125-133) & $\begin{array}{l}\text { ICS } \\
\text { IFN- } \gamma\end{array}$ & 52 \\
\hline
\end{tabular}

DC, dendritic cell; ICS, intracellular cytokine staining; IFN- $\gamma$, interferon- $\gamma$.

The table shows the autoantigens and the derived peptides identified in our samples as well as the preparation in which we could identify them, the autoimmune disease in which T-cell responses against the autoantigen/epitope have been experimentally verified, the epitope (where available) as well as the experimental method by which T-cell responses were measured in the respective publication. 
example is p53, a tumour suppressor with a central role in tumour development. Here, we detected a p53-derived peptide presented on MHC I on DCs (Supplementary Data 1 and 2), which should lead to thymic deletion of p53-specific CD8 ${ }^{+} \mathrm{T}$ cells. This is in line with a recent ovarian cancer study describing the lack of spontaneous p53-specific $\mathrm{CD}^{+}$, but not $\mathrm{CD} 4^{+}{ }_{-}$ specific $\mathrm{T}$-cell responses in healthy donors and patients with high antibody titres against p53 (ref. 43).

\section{Discussion}

In summary, we provide here the first characterization of the peptide repertoire presented by MHC molecules in the healthy human thymus in vivo with a focus on those epitopes presented by $\mathrm{mDCs}$ that are important for negative selection. Interestingly, both known autoantigens as well as tumour-associated antigens were detected. We observed a high degree of inter-individual variability between donors that can in part be explained by differences in HLA type but might also be partially due to differences in protein expression. Furthermore, mDCs and nonmDC APCs present different, only partially overlapping sets of peptides, probably due to differences both in self-protein expression as well as differences in their antigen-processing machinery ${ }^{13,15}$.

Although our data contribute to answer the long-standing question of which peptides are physiologically involved in T-cell selection in the human thymus, many issues still remain to be addressed before we fully understand the process of central tolerance. For example, analysis of ligands derived from isolated mTECs or cTECs as well as low abundance ligands presented by DCs is currently limited by available technology. For this reason, the data presented here are from mDCs only, and do not include ligands from isolated mTECs, which also contribute to central tolerance. Another question that remains to be addressed is whether the thymic ligandome changes considerably during the lifetime of an individual. For obvious reasons it will be difficult to answer this question experimentally in humans, but some previous studies suggest that variation is likely to occur. Thymic DCs and mTECs have a half-life of about 2-3 weeks and at least for mTECs, considerable differences in tissue-restricted antigen expression between different cells have been described ${ }^{44}$. Our and other data support the idea that thymic DCs sample the blood stream for proteins, and peripheral DCs can take up antigens, migrate to the thymus and delete the corresponding $\mathrm{T}$ cells $\mathrm{s}^{8,12}$. Changes in the periphery would therefore be expected to impact on the peptides presented in the thymus.

Our data should pave the way for a comprehensive understanding of central tolerance and might one day help to improve the design of therapeutic interventions such as cancer vaccines. The expression of ligands derived from tumour-associated antigens on thymic APCs highlights the need for a more systematic analysis of potential cancer vaccine candidates, employing our data to preselect or exclude antigens from the growing list of candidates in future vaccine trials.

\footnotetext{
Methods

Thymic samples. Normal human thymus samples were obtained from children undergoing corrective cardiac surgery (Department of Cardiac Surgery, University of Tübingen, Germany) after parents' informed consent and approval by the Institutional Review Board of the Medical Faculty of the University of Tübingen. For details of donors see Table 3 .
}

Genotyping of thymic tissues. DNA for HLA genotyping was extracted from thymus tissue using the QIAamp DNA Blood Mini kit (Qiagen). Low-resolution HLA typing was performed by the Institute for Clinical and Experimental Transfusion Medicine (IKET), University of Tübingen, Germany.

\section{Table 3 | Age, gender and HLA typing of thymus tissue} donors included in this study.

\begin{tabular}{llcl} 
Tissue no. & Age & Gender & HLA typing \\
\hline TT208 & 15 years & $F$ & $A^{\star} 01, A^{\star} 33, B^{\star} 49: 01, B^{\star} 51, C^{\star} 07, C^{\star} 16$ \\
& & & $D R B 1^{\star} 11, D R B 1^{\star} 13, D R B 3$
\end{tabular}

\begin{tabular}{|c|c|c|c|}
\hline TT222 & 6 months & $F$ & $\begin{array}{l}A^{\star} 01, A^{\star} 24, B^{\star} 40, B^{\star} 44 \\
D^{2} B 1^{\star} 04, D R B 1^{\star} 12, D R B 3, D R B 4\end{array}$ \\
\hline TT233 & 6 years & $\mathrm{F}$ & $\begin{array}{l}A^{\star} 02, B^{\star} 78, C^{\star} 16 \\
D^{*} 11^{\star} 13 \text { DRB3 }\end{array}$ \\
\hline TT246 & 2 years & M & $\begin{array}{l}A^{\star} 02, A^{\star} 25, B^{\star} 13, B^{\star} 18, C^{\star} 06, C^{\star} 12 \\
D R B 1^{\star} 07: 01, D R B 11^{\star} 15: 01\end{array}$ \\
\hline TT247 & 2 years & M & $\begin{array}{l}A^{\star} 01, A^{\star} 30, B^{\star} 08, B^{\star} 27 \\
D R B 1^{\star} 03: 01, D R B 1^{\star} 15: 01\end{array}$ \\
\hline TT251 & 8 months & M & $\begin{array}{l}A^{\star} 26, A^{\star} 66, B^{\star} 35, B^{\star} 38, C^{\star} 04, C^{\star} 12 \\
D^{2} B 1^{\star} 03, D R 1^{*} 11, D R B 3\end{array}$ \\
\hline ТT259 & 5 years & M & $\begin{array}{l}\mathrm{A}^{\star} 02, \mathrm{~A}^{\star} 24, \mathrm{~B}^{\star} 13, \mathrm{~B}^{\star} 27, \mathrm{C}^{\star} 01, \mathrm{C}^{\star} 06 \\
\mathrm{DRB}^{\star} 0103, \mathrm{DRB} 1^{\star} 15, \mathrm{DRB} 5\end{array}$ \\
\hline ТT260 & 11 months & $\mathrm{F}$ & $\begin{array}{l}A^{\star} 24, A^{\star} 26, B^{\star} 18, B^{\star} 55, C^{\star} 02, C^{\star} 03 \\
D R B 1^{\star} 11, D R B 3\end{array}$ \\
\hline
\end{tabular}

F, female; HLA, human leucocyte antigen; $M$, male

Isolation of thymic DCs. mDCs were isolated from thymus tissue after mechanical and enzymatic disruption (collagenase $\left(1 \mathrm{mg} \mathrm{ml}^{-1}\right)$ and DNase $\left(50 \mu \mathrm{g} \mathrm{ml}^{-1}\right.$, both from Roche), at $37^{\circ} \mathrm{C}$, two to three times for 30-45 min $)^{14}$. APCs were enriched from the resulting single-cell suspension by Percoll gradient centrifugation (density: $1.07 \mathrm{~g} \mathrm{ml}^{-1}, 30 \mathrm{~min}, 3,500 \mathrm{~g}, 4^{\circ} \mathrm{C}$ ) and DCs were isolated by magnetic beads (CD11c-PE and anti-PE beads, Miltenyi Biotec) from the low-density fraction following the manufacturer's instructions. Both the positive (DC) and negative (DC-depleted) fractions (for composition see Supplementary Table S1 and Supplementary Fig. S1) were collected. A summary of the workflow is given in Fig. 1.

FACS Analysis. Single-cell suspensions from whole thymus, the APC-enriched fraction after Percoll, DC and DC-depleted fractions were stained with antibodies against HLA-DR (PerCP, 307628, 1:50), CD19 (FITC, 302205, 1:20), CD14 (APC 301807, 1:50), CD45RA (FITC, 304105, 1:10), CD123 (PE/Cy7, 306009, 1:10), (all from Biolegend), CD45 (PB, PB986, 1:10, DAKO), BDCA-4 (PE, 130-090-533, 1:10), CD11c (PE, 130-092-411, 1:10), EpCAM (APC, 130-091-254, 1:10, from Miltenyi Biotec), CDR2 (1:50, Bruno Kyewski, DKFZ, Heidelberg; labelled with Alexa488 using the Alexa Fluor 488 Protein Labelling kit from Molecular Probes according to manufacturer's instructions). mDCs were gated as $\mathrm{CD} 11 \mathrm{c}^{+}, \mathrm{B}$ cells were gated as $\mathrm{CD} 19^{+}$, pDCs as CD45RA ${ }^{+} \mathrm{BDCA}_{-} 4^{+}$, monocytes as CD14 ${ }^{+}$, mTEC and cTEC as CD $45^{\text {lo-neg }}$ EpCAM $^{\text {hi }}$ or CD $45^{\text {lo-neg }}$ EpCAM $^{\text {lo } C D R 2 ~}{ }^{+}$, respectively.

Elution of MHC ligands. MHC-bound peptides were isolated using antibodies L234 (HLA-DR) and W6/32 (pan-HLA class I) ${ }^{45,46}$. We have also published a detailed step-by-step protocol of this procedure ${ }^{45}$. Frozen-cell pellets or tissue pieces were lysed with $0.6 \%$ CHAPS with protease inhibitors in PBS, stirring for $2 \mathrm{~h}$ at $4{ }^{\circ} \mathrm{C}$. The resulting lysate was ultrasonicated, and debris pelleted by centrifugation $(4,000 \mathrm{~g}$ for $20 \mathrm{~min}$, followed by ultracentrifugation at 40,000 r.p.m. for $1 \mathrm{~h})$. The supernatant was clarified by ultrafiltration $(0.2 \mu \mathrm{m}$ pore size, Sartolab $\mathrm{P}$ plus, Sartorius) and peptide-MHC complexes were isolated from the supernatant by affinity chromatography (antibodies L234 (MHC II) or W6/32 (MHC I) coupled to $\mathrm{CNBr}$-Sepharose, overnight, $4^{\circ} \mathrm{C}$ ). Bound $\mathrm{MHC}$-peptide complexes were eluted with $0.1 \%$ TFA. Eluted peptides were separated from MHC proteins by centrifugation though a filter with a MW cutoff of $10 \mathrm{kDa}$ and analysed by LC-MS/MS.

Identification of MHC ligands. Nanoscale liquid chromatography of MHC ligands was performed with a Waters NanoAcquity UPLC system equipped with a $75 \mu \mathrm{m} \times 150 \mathrm{~mm}$ BEH C18 reversed phase column and a $2.6-\mu$ PEEKSIL-sample loop (SGE, Darmstadt, Germany). The aqueous mobile phase (mobile phase A) was $\mathrm{H}_{2} \mathrm{O}$ with $0.1 \%$ formic acid. The organic mobile phase (mobile phase $\mathrm{B}$ ) was $0.1 \%$ 
formic acid in acetonitrile. Samples ( $2.6 \mu \mathrm{l}$ injection) were loaded onto the column in direct injection mode with $3 \%$ mobile phase B for 15 min at $400 \mathrm{nlmin}^{-1}$, followed by an additional $10 \mathrm{~min}$ wash $(3 \% \mathrm{~B})$ for $10 \mathrm{~min}$ at $300 \mathrm{nl} \mathrm{min}^{-1}$. Peptides were eluted from the column with a gradient from $3-35 \%$ mobile phase B over $120 \mathrm{~min}$ at $300 \mathrm{nl} \mathrm{min}^{-1}$ followed by a 20 -min rinse of $80 \%$ mobile phase B. The column was immediately re-equilibrated at initial conditions ( $3 \%$ mobile phase B)

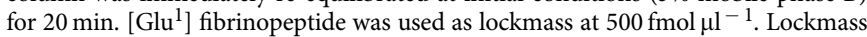
solution was delivered from the auxiliary pump of the NanoAcquity system at $400 \mathrm{nl} \mathrm{min}^{-1}$ to the reference sprayer of the NanoLockSpray source. MS/MS analysis of peptides was performed by using a Waters Q-Tof Premier mass spectrometer operated in $\mathrm{V}$-mode with typical resolving power of $R=10.000$ in datadependent acquisition mode, selecting the three most intense precursor ions for fractionation in the collision cell. Fragmentation of the parent ions was achieved by collision with argon atoms. Collision energy was varied from $15-35 \mathrm{eV}$ dependent on precursor ion mass and charge. The integration time for the TOF analyser was $1 \mathrm{~s}$ with an interscan delay of $0.1 \mathrm{~s}$.

Interpretation of MS/MS fragmentation spectra was carried out manually using MassLynx 4.0 software ${ }^{47}$. For manual peptide identification, sequence tag searches were done using Mascot 2.0 software (peptide and MS/MS tolerance, $0.2 \mathrm{Da}$; National Center for Biotechnology Information non-redundant (NCBInr) database updated monthly and restricted for search to human entries), and relevant hits were assessed manually and not by evaluation of the Mascot score. Criteria for manual identification were: a reasonable interpretation of at least $95 \%$ of all fragment peaks, complete sequence coverage with MS/MS fragments, and signal intensities of fragment ion peaks that match breakage probabilities of the respective sequence.

The obtained peptide sequences were assigned to source proteins using the BLAST algorithm and further bioinformatics analysis ${ }^{48,49}$ was performed. The MHC class I and MHC class II ligands in different thymic cell preparations were assigned to subcellular compartments or functional categories according to Gene Ontology annotations (www.geneontology.org). In cases where this search yielded no or incomplete information, annotation was performed manually based on information from WoLF PSORT ${ }^{48}$ (wolfpsort.org), SubLoc ${ }^{49}$

(www.bioinfo.tsinghua.edu.cn/SubLoc/), SwissProt and published literature. Source proteins were then grouped to the classical MHC I (cytosolic, nuclear) or MHC II pathway (extracellular, vesicular and cell membrane), the mitochondrial or ER compartment. To facilitate interpretation of data for biological function, related subcategories were grouped together, for example, 'signalling', 'cell-cell signalling' and 'signal transduction' were grouped together as 'signalling'. All peptides were assigned to a specific HLA allotype by taking the HLA type of the patient into consideration as well as known binding motifs of different allotypes ${ }^{50}$. Peptides were assigned to the presenting HLA molecule by comparison of their anchor amino acids with known HLA allele-specific peptide motifs. In order to carry out an automated, reproducible assignment to the correct HLA allotype, all peptide sequences were subjected to epitope prediction using the database SYFPEITHI (ref. 50) (www.syfpeithi.de), evaluating the preferential binding to one of the HLA allotypes defined by HLA typing. SYFPEITHI calculates scores according to the preferred amino acids in each sequence position. Peptides receiving more than the half-maximal score for a given HLA molecule were assigned to the respective molecule. It has to be noted that ligands of different HLA molecules may achieve different maximal scores. The maximal scores are 44 for HLA-A ${ }^{*} 03,44$ for HLA-A ${ }^{\star} 26,29$ for HLA-A ${ }^{\star} 31,37$ for HLA-A ${ }^{\star} 32,33$ for HLA-B ${ }^{\star} 07,30$ for HLA-B ${ }^{\star} 38$ and 38 for HLA-B ${ }^{\star} 51$. If epitope prediction was not possible by the web version of SYFPEITHI, an in-house version with extended options was used. This was the case for predictions of HLA-A ${ }^{\star} 31$ and HLA-A $A^{\star} 32$ ligands and for rarely occurring peptide lengths (8 mer, 11 mer) of other HLA allotypes.

Reproducibility analysis. For method validation and reproducibility analysis, MHC I peptides from PBMC of healthy volunteers were isolated and technical replicates performed. For statistical analysis of the intra-sample (that is, technical) reproducibility, the overlaps of every conceivable pair of technical replicates were calculated for one randomly selected sample. For the assessment of inter-individual variability we analysed the overlaps of HLA ligand source proteomes of five healthy volunteer PBMC samples. We performed comprehensive overlap analysis of all five samples. For the statistical analysis of inter-individual variability, paired overlap analyses of all 10 possible combinations of source proteomes were performed. The two populations (technical replicate overlaps versus inter-individual overlaps) were compared using a two-sided, unpaired Student's $t$-test not assuming equal standard deviations (Supplementary Fig. S3).

\section{References}

1. Kyewski, B. \& Klein, L. A central role for central tolerance. Annu. Rev. Immunol. 24, 571-606 (2006).

2. Klein, L., Hinterberger, M., Wirnsberger, G. \& Kyewski, B. Antigen presentation in the thymus for positive selection and central tolerance induction. Nat. Rev. Immunol. 9, 833-844 (2009).

3. Liu, G. Y. et al. Low avidity recognition of self-antigen by $\mathrm{T}$ cells permits escape from central tolerance. Immunity 3, 407-415 (1995).
4. Mazda, O., Watanabe, Y., Gyotoku, J. \& Katsura, Y. Requirement of dendritic cells and B cells in the clonal deletion of Mls-reactive T cells in the thymus. J. Exp. Med. 173, 539-547 (1991).

5. Brocker, T., Riedinger, M. \& Karjalainen, K. Targeted expression of major histocompatibility complex (MHC) class II molecules demonstrates that dendritic cells can induce negative but not positive selection of thymocytes in vivo. J. Exp. Med. 185, 541-550 (1997).

6. Hubert, F. X. et al. Aire regulates the transfer of antigen from mTECs to dendritic cells for induction of thymic tolerance. Blood 118, 2462-2472 (2011).

7. Gallegos, A. M. \& Bevan, M. J. Central tolerance to tissue-specific antigens mediated by direct and indirect antigen presentation. J. Exp. Med. 200, 1039-1049 (2004).

8. Hadeiba, H. et al. Plasmacytoid dendritic cells transport peripheral antigens to the thymus to promote central tolerance. Immunity 36, 438-450 (2012).

9. Kleindienst, P., Chretien, I., Winkler, T. \& Brocker, T. Functional comparison of thymic B cells and dendritic cells in vivo. Blood 95, 2610-2616 (2000).

10. Abramson, J., Giraud, M., Benoist, C. \& Mathis, D. Aire's partners in the molecular control of immunological tolerance. Cell 140, 123-135 (2010).

11. Derbinski, J., Schulte, A., Kyewski, B. \& Klein, L. Promiscuous gene expression in medullary thymic epithelial cells mirrors the peripheral self. Nat. Immunol. 2, 1032-1039 (2001).

12. Bonasio, R. et al. Clonal deletion of thymocytes by circulating dendritic cells homing to the thymus. Nat. Immunol. 7, 1092-1100 (2006).

13. Murata, S. et al. Regulation of CD $8+\mathrm{T}$ cell development by thymus-specific proteasomes. Science 316, 1349-1353 (2007).

14. Nakagawa, T. et al. Cathepsin L: critical role in Ii degradation and CD4 T cell selection in the thymus. Science 280, 450-453 (1998).

15. Stoeckle, C. et al. Cathepsin S dominates autoantigen processing in human thymic dendritic cells. J. Autoimmun. 38, 332-343 (2012).

16. Tolosa, E. et al. Cathepsin V is involved in the degradation of invariant chain in human thymus and is overexpressed in myasthenia gravis. J. Clin. Invest. 112, 517-526 (2003).

17. Marrack, P., Ignatowicz, L., Kappler, J. W., Boymel, J. \& Freed, J. H. Comparison of peptides bound to spleen and thymus class II. J. Exp. Med. 178, 2173-2183 (1993).

18. Santori, F. R. et al. Rare, structurally homologous self-peptides promote thymocyte positive selection. Immunity 17, 131-142 (2002).

19. Hogquist, K. A. et al. Identification of a naturally occurring ligand for thymic positive selection. Immunity 6, 389-399 (1997).

20. Fortier, M. H. et al. The MHC class I peptide repertoire is molded by the transcriptome. J. Exp. Med. 205, 595-610 (2008).

21. Garcia, C. A. et al. Dendritic cells in human thymus and periphery display a proinsulin epitope in a transcription-dependent, capture-independent fashion. J. Immunol. 175, 2111-2122 (2005).

22. Villadangos, J. A. \& Schnorrer, P Intrinsic and cooperative antigen-presenting functions of dendritic-cell subsets in vivo. Nat. Rev. Immunol. 7, 543-555 (2007).

23. Schmid, D., Pypaert, M. \& Munz, C. Antigen-loading compartments for major histocompatibility complex class II molecules continuously receive input from autophagosomes. Immunity 26, 79-92 (2007).

24. Dengjel, J. et al. Autophagy promotes MHC class II presentation of peptides from intracellular source proteins. Proc. Natl Acad. Sci. USA 102, 7922-7927 (2005).

25. Koble, C. \& Kyewski, B. The thymic medulla: a unique microenvironment for intercellular self-antigen transfer. J. Exp. Med. 206, 1505-1513 (2009).

26. Klein, L., Hinterberger, M., von Rohrscheidt, J. \& Aichinger, M. Autonomous versus dendritic cell-dependent contributions of medullary thymic epithelial cells to central tolerance. Trends Immunol. 32, 188-193 (2011).

27. Nedjic, J., Aichinger, M., Emmerich, J., Mizushima, N. \& Klein, L. Autophagy in thymic epithelium shapes the T-cell repertoire and is essential for tolerance. Nature 455, 396-400 (2008).

28. Gray, D., Abramson, J., Benoist, C. \& Mathis, D. Proliferative arrest and rapid turnover of thymic epithelial cells expressing Aire. J. Exp. Med. 204, 2521-2528 (2007).

29. Thery, C. et al. Indirect activation of naive CD4 $+\mathrm{T}$ cells by dendritic cell-derived exosomes. Nat. Immunol. 3, 1156-1162 (2002).

30. Millet, V., Naquet, P. \& Guinamard, R. R. Intercellular MHC transfer between thymic epithelial and dendritic cells. Eur. J. Immunol. 38, 1257-1263 (2008).

31. Munz, C. Enhancing immunity through autophagy. Annu. Rev. Immunol. 27, 423-449 (2009).

32. Dongre, A. R. et al. In vivo MHC class II presentation of cytosolic proteins revealed by rapid automated tandem mass spectrometry and functional analyses. Eur. J. Immunol. 31, 1485-1494 (2001).

33. Hogquist, K. A., Baldwin, T. A. \& Jameson, S. C. Central tolerance: learning self-control in the thymus. Nat. Rev. Immunol. 5, 772-782 (2005).

34. Feitsma, A. L. et al. Identification of citrullinated vimentin peptides as T cell epitopes in HLA-DR4-positive patients with rheumatoid arthritis. Arthritis Rheum. 62, 117-125 (2010). 
35. Wahlstrom, J. et al. Identification of HLA-DR-bound peptides presented by human bronchoalveolar lavage cells in sarcoidosis. J. Clin. Invest. 117, 3576-3582 (2007).

36. Forooghian, F., Cheung, R. K., Smith, W. C., O'Connor, P. \& Dosch, H. M. Enolase and arrestin are novel nonmyelin autoantigens in multiple sclerosis. J. Clin. Immunol. 27, 388-396 (2007).

37. Abulafia-Lapid, R., Gillis, D., Yosef, O., Atlan, H. \& Cohen, I. R. T cells and autoantibodies to human HSP70 in type 1 diabetes in children. J. Autoimmun. 20, 313-321 (2003).

38. Gotter, J., Brors, B., Hergenhahn, M. \& Kyewski, B. Medullary epithelial cells of the human thymus express a highly diverse selection of tissue-specific genes colocalized in chromosomal clusters. J. Exp. Med. 199, 155-166 (2004).

39. Bos, R. et al. Expression of a natural tumor antigen by thymic epithelial cells impairs the tumor-protective CD $4+$ T-cell repertoire. Cancer Res. 65, 6443-6449 (2005).

40. Kruger, T. et al. Lessons to be learned from primary renal cell carcinomas: novel tumor antigens and HLA ligands for immunotherapy. Cancer Immunol. Immunother. 54, 826-836 (2005).

41. Stickel, J. S. et al. HLA ligand profiles of primary renal cell carcinoma maintained in metastases. Cancer Immunol. Immunother. 58, 1407-1417 (2009).

42. Hernandez, J., Lee, P. P., Davis, M. M. \& Sherman, L. A. The use of HLA A2.1/ p53 peptide tetramers to visualize the impact of self tolerance on the TCR repertoire. J. Immunol. 164, 596-602 (2000).

43. Tsuji, T. et al. Split T cell tolerance against a self/tumor antigen: spontaneous CD4 + but not CD8 $+\mathrm{T}$ cell responses against p53 in cancer patients and healthy donors. PLoS One 6, e23651 (2011).

44. Taubert, R., Schwendemann, J. \& Kyewski, B. Highly variable expression of tissue-restricted self-antigens in human thymus: implications for self-tolerance and autoimmunity. Eur. J. Immunol. 37, 838-848 (2007).

45. Kowalewski, D. J. \& Stevanovic, S. Biochemical large-scale identification of MHC class I ligands. Methods Mol. Biol. 960, 145-157 (2013).

46. Falk, K., Rotzschke, O., Stevanovic, S., Jung, G. \& Rammensee, H. G. Allele-specific motifs revealed by sequencing of self-peptides eluted from MHC molecules. Nature 351, 290-296 (1991).

47. Weinzierl, A. O. et al. Distorted relation between mRNA copy number and corresponding major histocompatibility complex ligand density on the cell surface. Mol. Cell Proteomics 6, 102-113 (2007).

48. Horton, P. et al. WoLF PSORT: protein localization predictor. Nucleic Acids Res. 35, W585-W587 (2007).

49. Chen, H., Huang, N. \& Sun, Z. SubLoc: a server/client suite for protein subcellular location based on SOAP. Bioinformatics 22, 376-377 (2006).

50. Rammensee, H., Bachmann, J., Emmerich, N. P., Bachor, O. A. \& Stevanovic, S. SYFPEITHI: database for MHC ligands and peptide motifs. Immunogenetics 50, 213-219 (1999)
51. Wahlstrom, J. et al. Autoimmune T cell responses to antigenic peptides presented by bronchoalveolar lavage cell HLA-DR molecules in sarcoidosis. Clin. Immunol. 133, 353-363 (2009).

52. Johnston, A., Gudjonsson, J. E., Sigmundsdottir, H., Love, T. J. \& Valdimarsson $\mathrm{H}$. Peripheral blood T cell responses to keratin peptides that share sequences with streptococcal $\mathrm{M}$ proteins are largely restricted to skin-homing CD8( +) T cells. Clin. Exp. Immunol. 138, 83-93 (2004).

\section{Acknowledgements}

We thank the young patients and their parents for donating thymus samples, Professor Gerhard Ziemer, Professor Hermann Aebert, Dr Volker Steger and Dr Wilke Schneider (Tübingen, Germany) for providing us with the specimens, Professor Dorothee Wernet (Tübingen, Germany) for HLA typing, Simone Pöschel for technical assistance, Daniel Kowalewski (Tübingen, Germany) for methodological support and Professor Bruno Kyewski (Heidelberg, Germany) for the CDR2 antibody. This work was supported by the Deutsche Forschungsgemeinschaft (SFB 685, Graduate School 794, INST 371/23-1 FUGG), the Hertie Foundation, the BMBF (e:Bio Express2Present, 0316179C) and the Forschungszentrum Immunologie of the Johannes Gutenberg University Mainz.

\section{Author contributions}

C.S. designed the project with support from E.T. and coordinated research. C.S., E.A. and N.H. designed and performed experiments and de novo sequencing data analysis with P.K., S.T., I.R. and M.G. providing assistance. LC-MS/MS and bioinformatic analysis was carried out by S.T., S.S. provided valuable help with peptide HLA assignment and methodology, E.T., S.T. and H.S. contributed scientific discussion. A.M. coordinated thymi sample collection and contributed to the direction of the study. E.A. and C.S evaluated the data and wrote the manuscript, which was discussed, critically edited and approved by all co-authors. C.S. and A.M. share senior authorship.

\section{Additional information}

Supplementary Information accompanies this paper at http://www.nature.com/ naturecommunications

Competing financial interests: The authors declare no competing financial interests.

Reprints and permission information is available online at http://npg.nature.com/ reprintsandpermissions/

How to cite this article: Adamopoulou, E. et al. Exploring the MHC-peptide matrix of central tolerance in the human thymus. Nat. Commun. 4:2039 doi: 10.1038/ncomms3039 (2013).

(c) $\Theta$ This work is licensed under a Creative Commons AttributionCoY NC ND NonCommercial-NoDerivs 3.0 Unported License.
this license, visit http://creativecommons.org/licenses/by-nc-nd/3.0/ 